\title{
Constraints, limits and extensions for nuclear energy functionals
}

\author{
Gianluca Colò \\ Dipartimento di Fisica, Università degli Studi, and INFN, Sez. di Milano, via Celoria 16, 20133 Milano (Italy)
}

\begin{abstract}
In the present contribution, we discuss the behavior of Skyrme forces when they are employed to study both neutron stars and giant resonance states in ${ }^{208} \mathrm{~Pb}$ within the fully self-consistent Random Phase Approximation (RPA). We point out that clear correlations exist between the results for the isoscalar monopole and isovector dipole resonances (ISGMR and IVGDR), and definite quantities which characterize the equation of state (EOS) of uniform matter. We propose that the RPA results or, to some extent, the mentioned EOS parameters, are used as constraints when a force is fitted. This suggestion can be valid also when the fit of a more general energy density functional is envisaged. We use our considerations to select a limited number of Skyrme forces (10) out of a large sample of 78 interactions.
\end{abstract}

Keywords: HF-RPA; Density Functional; Giant Resonances; EOS and symmetry energy

PACS: $21.10 . \mathrm{Re}, 21.30 . \mathrm{Fe}, 21.60 . \mathrm{Jz}, 21.65 . \mathrm{Ef}$

\section{INTRODUCTION}

The quest for an accurate density functional for atomic nuclei lies at the forefront of nuclear structure research. Nuclei are strongly interacting many-body systems. Except for the lightest among them, namely those having mass number $A$ smaller than $\approx 10-15$ [1], trying to include explicitly all correlations in the wavefunction is not feasible. One is obliged to reduce the complexity of the wavefunction and to employ effective interactions $V_{\text {eff }}$. Since more than three decades, one of the most widely used approaches in nuclear physics is the so-called self-consistent mean-field (SCMF) approach. In it, starting from an effective Hamiltonian $H_{\text {eff }}=T+V_{\text {eff }}$ where $T$ is the kinetic energy, one calculates the total energy $E$ as the expectation value of that Hamiltonian over the most general Slater determinant $|\Phi\rangle$, i.e., over the most general one-body density $\rho$ which is compatible with the symmetries of the system under study. This defines an energy density functional $\mathscr{E}$, that is,

$$
\left\langle\Phi\left|H_{\mathrm{eff}}\right| \Phi\right\rangle=E[\rho]=\int d_{3} r \mathscr{E}(\rho) .
$$

By minimizing the total energy $E[\rho]$, one can derive the nuclear ground-state. In the simplest case, that is, in a system which is not superfluid, this is achieved by means of the Hartee-Fock (HF) equations. Among effective interactions, the zero-range forces of the Skyrme type or the finite-range Gogny interactions, are the most popular. Their parameters are fitted on a limited number of known properties (saturation of nuclear matter, energies and radii of few magic nuclei). The relativistic mean field (RMF) models share the same philosophy, that is, the number of parameters is comparable and they are fitted in a similar way. For a recent review about the mean-field methods, their advantages and the obvious limitations, one can refer to [2].

Recently, many authors have pointed out that the nuclear energy functional can be more general and not necessarily obtained from an effective Hamiltonian. There are groups, around the world, who are working intensively with the aim of writing directly the energy density functional with the most general structure compatible with symmetries, and of fitting the parameters at that level. The rationale behind this, is the guarantee that an exact functional exists (provided by the Hohenberg-Kohn theorem). An alternative strategy consists in trying to derive the energy functional from an underlying theory, whether Brückner-Hartree-Fock or Dirac-Brückner.

While these attempts are certainly of paramount importance, they cannot suceed without a physical guidance. So, there is still work to be done on the existing functionals, albeit limited in their structure and not derived from an underlying theory. One should

- remove the approximations, if any, which are still present in the calculations for the ground-state and the excited states within the SCMF implementations;

- provide a clear link between functional parameters and observables; 
- propose extensions of the existing functionals as much as this appears necessary to account for measured observables.

The basic issue is to define the observables. We mentioned above those associated with the ground-state: the total energy and the density with the quantities that can be derived like radii, quadrupole moments etc. Their relevance is undeniable, and at the same time they have been already much discussed (see, e.g., [3] for a discussion of functionals which are fitted to nuclear masses on a large scale). Much less attention is usually paid to excited states. However, there exist states, like the giant resonances, whose properties carry general and relevant nuclear structure information. Consequently, we focus in this contribution on elucidating the links between the giant resonance properties and specific features of the existing Skyrme functionals. We then exploit these links by proposing a selection of Skyrme forces; our first screening is actually based on neutron star properties, following closely the work of Ref. [4].

Our discussion is based on the assumption of a relationship between the parameters of a functional and the results obtained from specific calculations of the excitation modes (in the case under study, the giant resonances). If, as recalled above, the HF equations provide the nuclear ground-state by minimizing the total energy, the corresponding time-dependent (TD) equations describe the oscillations around that minimum. In the limit of small amplitude, the TDHF equations reduce to the equations of the so-called Random Phase Approximation (RPA). RPA is a suitable theory to describe the nuclear giant resonances (although it cannot account for their spreading width). In self-consistent RPA, the residual interaction is derived from the ground-state mean field. Therefore, the RPA results depend only on the parameters of the effective Hamiltonian. In this way, one is able to link these parameters with the giant resonance properties, or with specific EOS parameters as we discuss below. Within phenomenological RPA, based e.g. on a Woods-Saxon mean field and a residual interaction which is fitted ad hoc, it is impossible to establish these links.

Most of the existing nuclei are open-shell systems in which the pairing correlations are active. In this case, the HF framework can be extended and one introduces the Hartree-Fock-Bogoliubov (HFB) one, in which a Slater determinant of independent quasiparticles instead of independent particles, is assumed. The corrsponding linear response theory which describes the small oscillations is the quasiparticle RPA (QRPA). In these approaches, the Skyrme force must be supplemented by a pairing interaction which is not the focus of our present discussion. We point out, however, in what follows, that pairing can have an effect also on high-lying states like giant resonances and impact our discussion of the relationships between the parameters of a Skyrme functional and the EOS quantities.

\section{SKETCH OF THE METHOD TO SOLVE THE (Q)RPA EQUATIONS}

We have at our disposal a fully self-consistent scheme for both RPA and QRPA. Some results from RPA have been first presented in Ref. [5]. Skyrme-RPA theory is well known since many years, especially in its matrix formulation. In our scheme, we first solve the HF equations in coordinate space and calculate the unoccupied states by using the resulting mean field and box boundary conditions (which means that the continuum is discretized). We build a basis of particle-hole (p-h) configurations and we diagonalize the associated RPA matrix, by checking carefully that the basis is large enough so that our results are stable. We should also mention that in our scheme there is no approximation in the residual interaction, in that all its terms are taken into account. In the calculations presented below, the box dimension is typically between $\approx 3-4$ times the size of the nucleus, and unoccupied states up to $\approx 60-80 \mathrm{MeV}$ are included in the model space.

The extension of our model for open-shell nuclei, in the form of a fully self-consistent QRPA based on HFB, has been presented for the first time in [6]. The formalism is analogous to that of Ref. [7]. The starting point is the solution of the HFB equations in coordinate space. In this case, the basis for QRPA is built using canonical states. This allows keeping the equations reasonably simple, that is, the part of the QRPA matrix associated with the residual interaction is the same as in the case of BCS (whereas the part associated with the unperturbed Hamiltonian has non-diagonal elements, since the canonical states are not eigenstates of that Hamiltonian). However, the price to be paid is that the canonical basis must be quite large (the energy cutoff is $\approx 150-200 \mathrm{MeV}$, at variance with the RPA case).

After solving the RPA or QRPA equations, we obtain the full set of eigenvalues and eigenvectors and from them the strength function $S(E)$ associated with, e.g., the IS monopole or IV dipole operators. The moments of the strength function are defined as $m_{k}=\int d E S(E) E^{k}$. The usual centroid energy is defined as $E_{0}=m_{1} / m_{0}$. In certain cases, and also in the discussion below, other definitions like $E_{-1}=\sqrt{m_{1} / m_{-1}}$ (called sometimes the constrained energy) are used. Obviously, all possible well-defined centroid energies coincide if the strength has a single, symmetric peak. In cases like the IS quadrupole, where there is a giant resonance but also a low-lying peak, centroid energies must be defined in a limited energy interval. 
We should point out that the quantity $E_{-1}=\sqrt{m_{1} / m_{-1}}$ could be obtained without resorting to a full QRPA calculation, at least in the nonrelativistic framework. In fact, $m_{1}$ can be obtained from the Thouless theorem and $m_{-1}$ from the dielectric theorem. These theorems, which have been known for long time in the non-superfluid case, have recently been demonstrated in the case with pairing - that is, in the case of self-consistent QRPA on top of HFB [8].

\section{REMINDER OF THE RELEVANT EOS PARAMETERS}

In Eq. (1) the energy density functional has been written in a schematic, oversimplified form. In fact, for systems that are not symmetric in neutrons and protons, the total energy must depend on both neutron and proton densities $\left(\rho_{q}\right.$, where $q$ labels $n, p)$. Moreover, in finite nuclei a local functional depends also on gradients of the densities, $\nabla \rho_{q}$, on kinetic energy densities, $\tau_{q}$, and on the so-called spin-orbit densities $J_{q}$ (for details, see Ref. [2]).

In uniform matter, only the dependence on spatial densities shows up. Instead of $\rho_{n}$ and $\rho_{p}$, one can employ as variables the total density $\rho$ and the local neutron-proton asymmetry, $\delta \equiv\left(\rho_{n}-\rho_{p}\right) / \rho$ (this quantity should not be confused with the global asymmetry $(N-Z) / A)$. In uniform asymmetric matter, we can further simplify $\mathscr{E}(\rho, \delta)$ by making a Taylor expansion in $\delta$ and retaining only the quadratic term,

$$
\mathscr{E}(\rho, \delta) \approx \mathscr{E}_{0}(\rho, \delta=0)+\mathscr{E}_{\text {sym }}(\rho) \delta^{2}=\mathscr{E}_{0}(\rho, \delta=0)+\rho S(\rho) \delta^{2} .
$$

It has been checked that the quartic term is negligible, for Skyrme functionals, at the densities of interest for our discussion [9, 10].

The first term at the r.h.s. of Eq. (2) is the energy density of symmetric nuclear matter; for it, the minimum of the energy per particle $E / A=\mathscr{E} / \rho$ is well known and used when functionals are fitted. The curvature around this minimum is simply related to the nuclear matter incompressibility, which reads

$$
K_{\infty}=\left.9 \rho_{0}^{2} \frac{d^{2}}{d \rho^{2}} \frac{\mathscr{E}_{0}}{\rho}\right|_{\rho=\rho_{0}}
$$

The second term at the r.h.s. of Eq. (2) defines the symmetry energy $S(\rho)$. This quantity, and in particular its density dependence, is presently much under debate and different contributions in the present volume deal with its determination, in keeping with its ubiquitous relevance in nuclear structure, heavy-ion reactions, and nuclear astrophysics. The density dependence of the symmetry energy around the saturation density $\rho_{0}$ of symmetric nuclear matter can be expressed by means of

$$
S(\rho)=S\left(\rho_{0}\right)+S^{\prime}\left(\rho_{0}\right)\left(\rho-\rho_{0}\right)+\frac{1}{2} S^{\prime \prime}\left(\rho-\rho_{0}\right)^{2}+\ldots
$$

Usually, one defines $S^{\prime}\left(\rho_{0}\right)=L / 3 \rho_{0}$ and $S^{\prime \prime}=K_{\text {sym }} / 9 \rho_{0}^{2}$; in fact, if $x=\frac{\rho-\rho_{0}}{3 \rho_{0}}, L$ and $K_{\text {sym }}$ are respectively $\frac{d S}{d x}$ and $\frac{d^{2} S}{d x^{2}}$.

Starting from a different point of view, we can relate the ISGMR energy $E_{I S G M R}$ in a given nucleus to the so-called finite nucleus incompressibility $K_{\mathrm{A}}$ which has been introduced in Ref. [11],

$$
K_{\mathrm{A}}=\frac{m\left\langle r^{2}\right\rangle_{0} E_{I S G M R}^{2}}{\hbar^{2}}
$$

(where $m$ is the nucleon mass and $\left\langle r^{2}\right\rangle_{0}$ is the ground-state expectation value). The interest of this quantity stems from the fact that if we consider a local functional like Skyrme written for a spherical system, and we calculate its second derivative around the minimum using various simplifying hypotheses, the main one being the use of the so-called "scaling model", we can write $K_{\mathrm{A}}$ in a form analogous to that of the mass formula, namely [11]

$$
K_{\mathrm{A}}=K_{\infty}+K_{\text {surf }} A^{-1 / 3}+K_{\tau}\left(\frac{N-Z}{A}\right)^{2}+K_{\text {Coul }} \frac{Z^{2}}{\mathrm{~A}^{4 / 3}} .
$$

Moreover, we can show that

$$
K_{\tau}=K_{\text {sym }}+3 L-\left.\frac{27 \rho_{0}^{2} L}{K_{\infty}} \frac{d^{3} \mathscr{E}}{d \rho^{3}}\right|_{\rho_{0}} .
$$

The last formula shows that a constraint on $K_{\tau}$ is reflected directly on the parameters which are associated with the density dependence of the symmetry energy, $L$ and $K_{\text {sym }}$. 


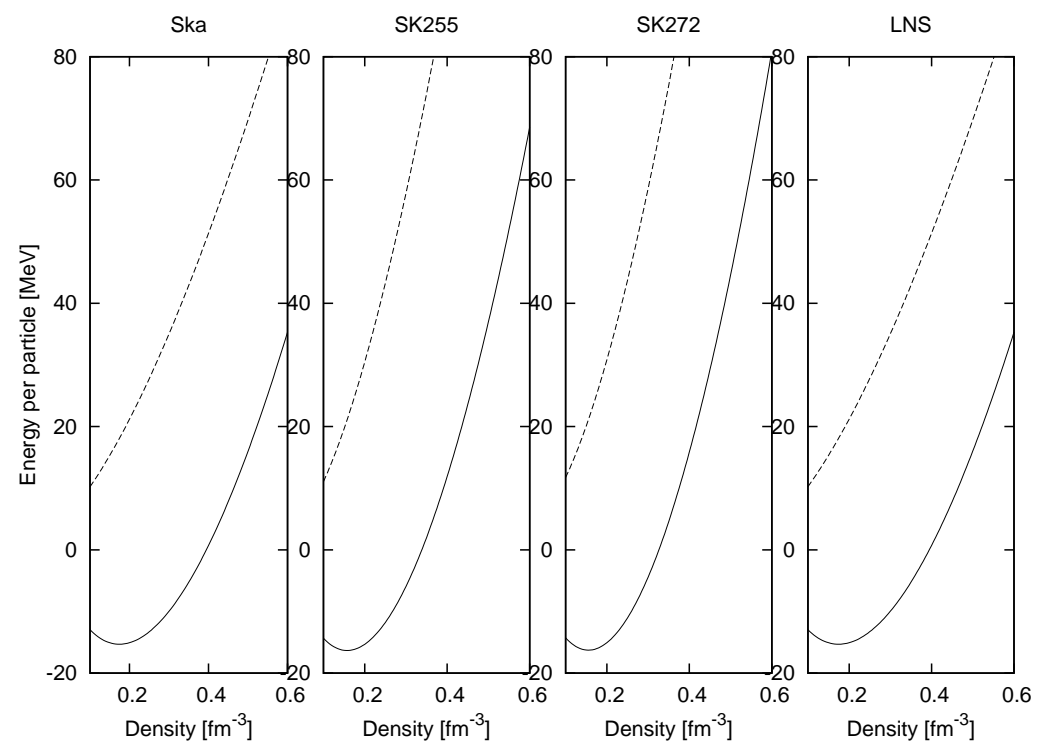

FIGURE 1. For the four forces which label the panels (they are Skyrme sets which have been not studied in Ref. [4]), we display the energy per particle in symmetric nuclear matter (full line) and in pure neutron matter (dashed line). Because of the fact that these quantities have a pronounced increase as a function of the density, and that the dashed curve never falls below the other one, these forces belong to the group I defined in [4] and lead to qualitatively correct neutron star properties.

\section{SKYRME SETS APPLIED TO NEUTRON STARS AND TO GIANT RESONANCES}

The usual complain concerning Skyrme functionals is that there exist too many different parametrizations, and it is true that probably around 100 or more parameter sets have been introduced in the literature. It should be stressed that not all of them are to be put on the same footing: whereas some sets have been used for long time after they have been first proposed, other sets are "marginal" in the sense that they have been fitted with very specific purposes and adopted only for one or few applications. We wish to propose a strategy to limit the number of Skyrme parameter sets to be considered "reasonable"; the hope is that the criteria we propose, eventually improved, can be used when the fitting of a universal functional [12] is envisaged.

The strategy we propose is applied to an ensemble of 78 Skyrme forces which is certainly large enough to demonstrate the effectiveness of the method. It is impossible to recover the parameters of all Skyrme sets ever introduced, and this effort would be meaningless since in principle many more sets can be produced. We start from the work already done in Ref. [4]: here, the authors consider as a starting point an ensemble of 87 forces which can be considered, quoting their words, "a representative sample of the Skyrme interactions used in the nuclear physics applications". These forces are reported in Table I of [4] and the reader can find there the original references, that we do not report here for the sake of brevity. Our starting sample is rather similar: we exclude, compare to [4] the sets SLy0, SLy1, SLy2, SLy3, SLy8, SLy9 (they are unpublished), SLy6, SLy7, SLy10 (they include the two-body center-of-mass correction), SkI1, SkI4, SkI6, SkO (they do not lead easily to convergent results), and we add the four forces Ska [13], SK255, SK272 [14] and LNS [15].

We apply the following criteria:

1. We select, among the 78 forces, those which have an overall satisfactory behavior as far as the density dependence of the symmetry energy in the range $0.1 \leq \rho \leq 0.6 \mathrm{fm}^{-3}$ is concerned (we follow closely Ref. [4] for this point).

2. For these forces we calculate the IVGDR in ${ }^{208} \mathrm{~Pb}$ and we make a further selection, by demanding that the forces reproduce the experimental value $E_{-1}=13.46 \mathrm{MeV}[16]$ within $\pm 1 \mathrm{MeV}$.

3. Finally, we also demand that the selected interactions reproduce the experimental value of $E_{-1}=14.17 \mathrm{MeV}$ for the ISGMR in ${ }^{208} \mathrm{~Pb}$ [17], with the same accuracy of $\pm 1 \mathrm{MeV}$.

The Skyrme sets which have "survived" this kind of selection will be listed at the end of our discussion. 


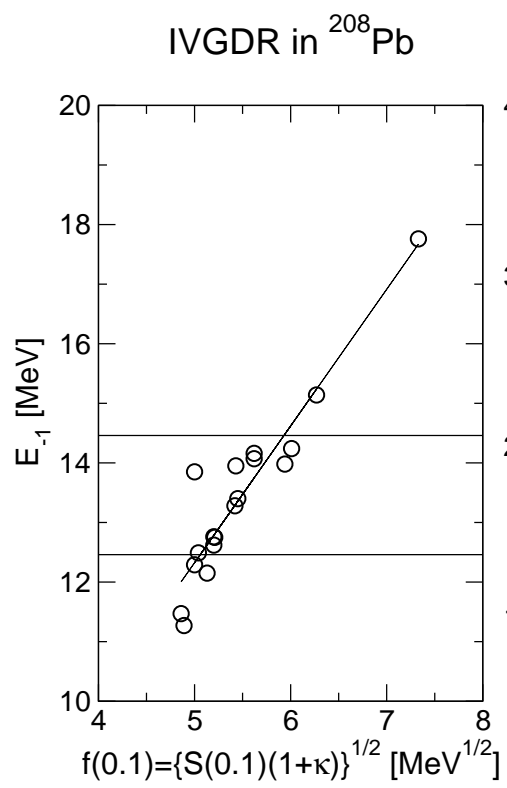

\section{Symmetry energy}

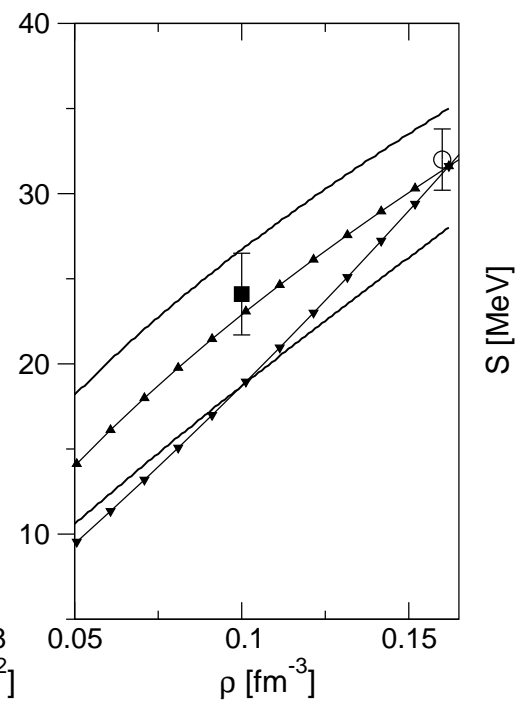

FIGURE 2. In the left panel, the dipole energy is displayed as a function of the quantity $f(0.1)$ defined in the text. The thin line is a linear fit while the horizontal full lines correspond to the experimental energy $\pm 1 \mathrm{MeV}$. In the right panel, the constraint on $S(0.1)$ extracted from the dipole is displayed with a black square and an associated error bar. The open circle (also with error bar) shows the constraint from Ref. [20]. The two thick lines, and the two lines which join small triangles, are bounds (upper and lower) for the symmetry energy $S(\rho)$ coming from the studies respectively of Refs. [22] and [23].

\section{Neutron stars and the overall behavior of the symmetry energy}

In Ref. [4] it has been tested whether Skyrme forces predict plausible neutron stars properties. The TolmanOppenheimer-Volkov (TOV) equation [18, 19] has been solved, coupled with the Skyrme EOS (supplemented by appropriate corrections for low densities), and the mass-radius relationship associated with a given parameter set has been given. The result is that Skyrme sets can be divided in three groups. Only the sets of group I reproduce the expected qualitative relationship: this has been found to be strictly related with the fact that the energy per particle increase quickly, as a function of density, both in symmetric nuclear matter and in pure neutron matter, with the latter being always characterized by a larger energy with respect to the former. We have checked the performance of the four sets that we have decided to add to the starting sample compared with [4]: in Fig. 1] we have displayed the mentioned quantities, so that it is clear that these interactions obey the conditions which are sufficient to be included in the group I of the satisfactory parameter sets. Since the energy in pure neutron matter equals the energy in symmetric nuclear matter plus $S$, we can say that in the proposed method of selection the overall behavior of the symmetry energy plays a key role.

In conclusion, after the selection we end up with 18 forces (Gs, Rs, SGI, SLy230a, SLy4, SLy5, SV, SkI2, SkI3, SkI5, SkMP, SkO', SkT4, SkT5, Ska, SK255, SK272, LNS).

\section{The IVGDR and the related constraint on the symmetry energy}

With the 18 selected forces we have calculated the energy of the IVGDR in ${ }^{208} \mathrm{~Pb}$. The results are displayed in the left panel of Fig. 2. where the energy $E_{-1}$ (cf. above) appears on the y-axis. On the x-axis the quantity

$$
f(0.1) \equiv \sqrt{S(0.1)(1+\kappa)}
$$

is shown, where $S(0.1)$ is the symmetry energy evaluated at $\rho=0.1 \mathrm{fm}^{-3}$ and $\kappa$ is the so-called enhancement factor of the IV dipole sum rule (with respect to the classical Thomas-Reiche-Kuhn sum rule). The quantity $f(0.1)$ has been already defined in [9], where physical arguments have been provided to justify a correlation between $f(0.1)$ and the dipole energy $E_{-1}$. We do not repeat these arguments here. We notice that the correlation is visible in the left panel 


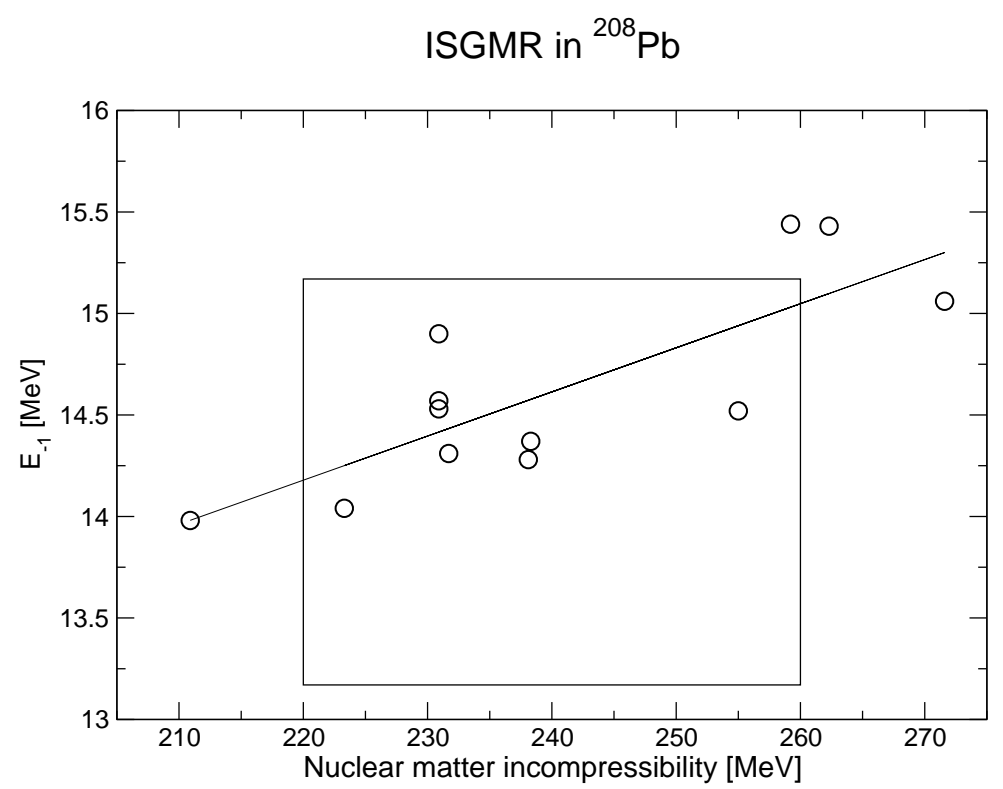

FIGURE 3. The monopole energy is displayed as a function of the nuclear matter incompressibility $K_{\infty}$ (cf. Eq. (3). The box defines a region which corresponds to $K_{\infty}=240 \pm 20 \mathrm{MeV}$ [26], and to a monopole energy which is within $\pm 1 \mathrm{MeV}$ with respect to the experimental value. See the discussion in the main text.

of Fig. 2 as testified by the thin line which corresponds to a linear fit. The two horizontal lines correspond to the experimental dipole energy $\pm 1 \mathrm{MeV}$ : the results associated with 12 forces (Gs, Rs, SGI, SLy230a, SLy4, SLy5, SkI3, SkMP, SkO', SK255, SK272 and LNS) lie within those lines, and these forces are selected for further considerations.

In Ref. [9] the correlation between the dipole energy and $f(0.1)$ has been used to extract a constraint on this latter quantity. Unfortunately, $f$ contains at the same time the symmetry energy as well as $\kappa$ and we miss an unambiguous experimental determination of the dipole enhancement factor. If one introduce an acceptable range for $\kappa$, between 0.18 and $0.26, S(0.1)$ is constrained in the interval $24.1 \pm 0.8 \mathrm{MeV}$. However, we have verified that in the ensemble of forces used in the present work (which includes some forces that were not considered in [9]), there are some which do reproduce the experimental IVGDR energy having $S(0.1)$ outside the range of $24.1 \pm 0.8 \mathrm{MeV}$. To account for this, we have displayed in the right panel of Fig. [2 the point corresponding to the dipole constraint with a larger error bar, numerically equal to $\pm 3 \sigma$ (that is, $24.1 \pm 2.4 \mathrm{MeV}$ ).

In the same panel, results from other groups are reported. In Ref. [20] the data on the Pygmy Dipole Resonance (PDR) obtained at GSI, Darmstadt has been compared with RMF calculations and eventually a range of values for $J$ has been extracted, which is shown in the panel with an open circle with its associated error bar $(J=32 \pm 1.8 \mathrm{MeV})$. In Ref. [21], a throughout analysis of nuclear surface symmetry energies has been carried out. This analysis led to $J=32.5 \pm 1 \mathrm{MeV}$ which is compatible with the previous value. We do not display the corresponding point in the figure, just to keep it reasonably clear and readable.

We also like to discuss, very briefly, the comparison with the constraints coming from studies of heavy-ion collisions. Data of isospin diffusion following the ${ }^{112} \mathrm{Sn}_{-}{ }^{124} \mathrm{Sn}$ reaction, have been anayzed using transport models, in particular the Improved Quantum Molecular Dynamics model (I) in [22] and the IBUU04 version of the Boltzmann-UehlingUhlenbeck (BUU) model (II) in [23]. We are not in the position to discuss merits and pitfalls of these models (for which we confer the reader to the original references). However, for each model we draw two curves which correspond to acceptable upper and lower limits. These are the full thick lines in the case of model (I) and the thin lines joining the triangles in the case of model (II).

In principle, other observables can be very effective to constrain the symmetry energy and its density dependence. We mention the sum rules of charge-exchange excitations [24], which have been measured yet with unsufficient precision, and the neutron radius of ${ }^{208} \mathrm{~Pb}$ [27], which should be very accurately determined by the PREX experiment. 

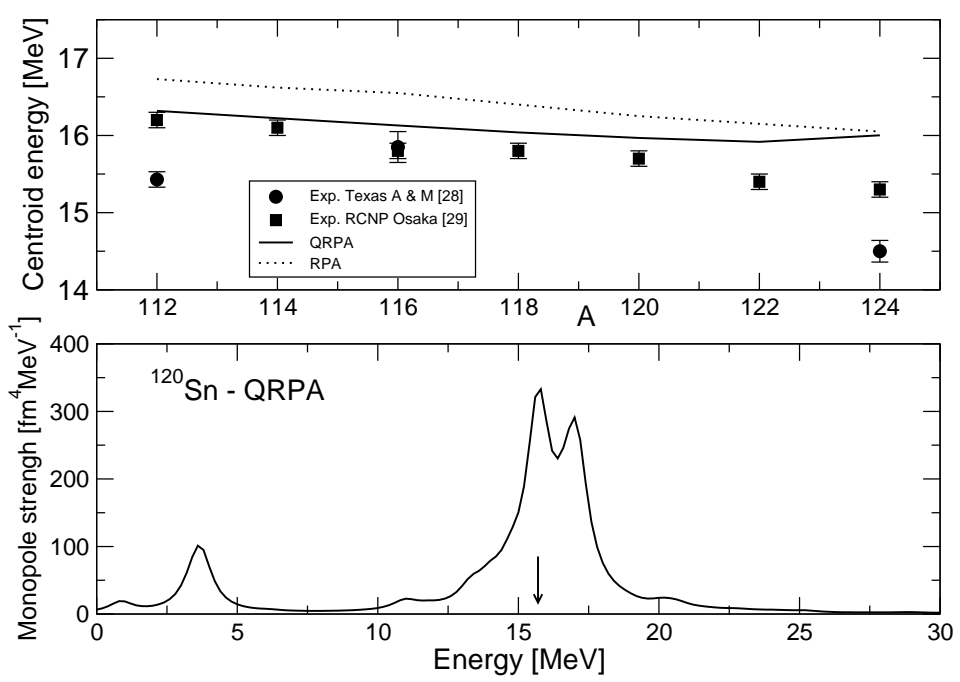

FIGURE 4. In the upper panel, the experimental results for the monopole centroid energies from [28] and [29] are displayed. They are compared with the RPA and QRPA results of Ref. [6]. In the lower panel, the QRPA strength distribution is shown in detail for the case of ${ }^{120} \mathrm{Sn}$. The discrete QRPA results have been smeared out with Lorentzians having $1 \mathrm{MeV}$ width, only for illustrative purposes. The arrow indcates the position of the experimental centroid from the upper panel.

\section{The ISGMR and the constraint on the nuclear incompressibility}

With the 12 forces mentioned in the previous subsection, we have calculated the ISGMR in ${ }^{208} \mathrm{~Pb}$. The result for the energy $E_{-1}$ is compared with the experimental finding, namely $14.17 \mathrm{MeV}$ [17]. Almost all the forces that have been selected through the calculation of the IVGDR, reproduce the ISGMR energy $\pm 1 \mathrm{MeV}$. Only SGI and SkI3 must be rejected, and we end up with a set of 10 forces, that is, Gs, Rs, SLy230a, SLy4, SLy5, SkMP, SkO', SK255, SK272 and LNS.

The results are shown in Fig. 3, as a function of the associated value of the nuclear matter incompressibility $K_{\infty}$ (cf. Eq. (3)). The thin line corresponds to a linear fit. The open box defines a region which corresponds to $K_{\infty}=240 \pm 20$ $\mathrm{MeV}$, and to a monopole energy which is within $\pm 1 \mathrm{MeV}$ with respect to the experimental value. The preferred range given by $K_{\infty}=240 \pm 20 \mathrm{MeV}$ has been extensively discussed in [26], and it actually comes from an analysis which includes not only Skyrme forces but a careful confrontation with results obtained with Gogny interactions and RMF parametrizations as well, so that we believe its validity is rather general. Also in Fig. 3, almost all the forces which reproduce reasonably the monopole energy, have an associated value of the incompressibility in that range (there are only two exceptions).

\section{Remarks on the ISGMR in open-shell isotopes}

As a side remark, albeit quite important, we discuss in this subsection the ISGMR in the Sn isotopes. In Ref. [27], the question has been raised "why is tin so soft" or, in other words, why do theoretical models (with a value of the incompressibility $K_{\infty}$ in the quoted range $K_{\infty}=240 \pm 20 \mathrm{MeV}$ ) that reproduce the values of the ISGMR energy in ${ }^{208} \mathrm{~Pb}$ as well as in ${ }^{90} \mathrm{Zr}$, tend to overestimate this energy in $\mathrm{Sn}$ isotopes ? These are semi-magic nuclei and neutrons are superfluid. Answering to the question above, implies among the rest a serious assessment of the effect of pairing correlations on the ISGMR.

This has motivated the work of Ref. [6], where a fully self-consistent QRPA based on HFB has been applied to the study of the monopole strength distribution in the Sn isotopes. The Skyrme force has been supplemented with an effective, zero-range, density-dependent pairing force. Three kinds of pairing forces have been tested, namely volume, surface, and mixed pairing forces. Writing the pairing force as

$$
V_{\text {pair }}\left(\mathbf{r}_{1}, \mathbf{r}_{2}\right)=V_{0}\left[1-\eta\left(\frac{\rho\left(\frac{\mathbf{r}_{1}+\mathbf{r}_{2}}{2}\right)}{\rho_{0}}\right)\right] \delta\left(\mathbf{r}_{1}-\mathbf{r}_{2}\right),
$$




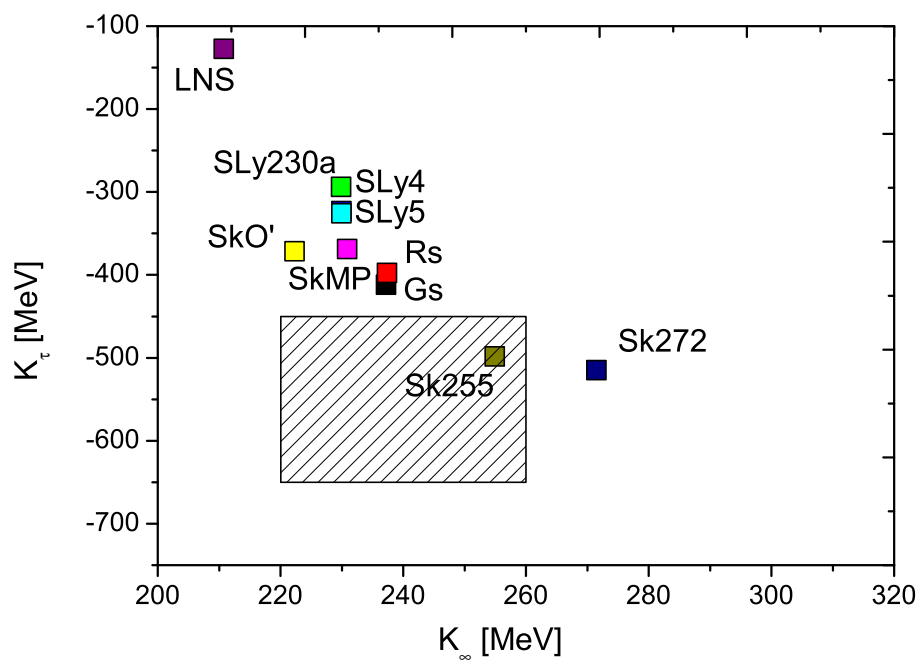

FIGURE 5. Values of $K_{\infty}$ and $K_{\tau}$ associated with the 10 Skyrme forces which have been selected. The relevance of the shaded box is discussed in the main text.

the three kinds of pairing correspond respectively to $\eta=0,1$ and $1 / 2 . \rho_{0}$ is fixed at $0.16 \mathrm{fm}^{-3}$ and the values of $V_{0}$ (for every kind of pairing but also for every Skyrme parameter set employed) are fixed by fitting the empirical value of the mean neutron gap of ${ }^{120} \mathrm{Sn}$. Pairing is treated consistently in HFB and QRPA, and also the (small) pairing rearrangement terms have been analyzed. The reader can consult [6] for further details.

Some of the main results of that investigation are in Fig. 4 Looking at the upper panel, one can notice a systematic shift downwards of the QRPA results with respect to RPA. This shift is due of the attractive monopole pairing matrix elements. It is not constant along the isotope chain, but it tends to decrease with increasing $N-Z$ : this effect has been explained in [6] as a consequence of the level occupancies. In the lower panel of Fig. (4) we show the QRPA monopole strength distribution in a typical case.

This particular set of results corresponds to the force $\mathrm{SkM}^{*}$ plus surface pairing. Since $\mathrm{SkM}^{*}$ has an associated value of $K_{\infty}$ given by $217 \mathrm{MeV}$, looking at ${ }^{112-120} \mathrm{Sn}$ one would conclude that the discrepancy between the values of the nuclear matter incompressibility extracted from the $\mathrm{Sn}$ isotopes and from ${ }^{208} \mathrm{~Pb}$ differ by about $10 \%$. Of course, this points to our still incomplete understanding of the details of the nuclear effective functionals - but the puzzle would be greater if the pairing contribution had been overlooked.

Still two considerations are in order. Firstly, in the two cases of ${ }^{112} \mathrm{Sn}$ and ${ }^{124} \mathrm{Sn}$, the results of the two experimental groups disagree quite seriously, but understanding the reasons of this discrepancy is in progress [30]. Secondly, the main motivation at the basis of the experiment reported in [29] was the extraction of the parameter $K_{\tau}$ defined by Eq. (6): in fact, the trend of the experimental data, plus the simplifying assumption which consists in setting $K_{\text {surf }} \approx-K_{\infty}$, have allowed extracting $K_{\tau}$ from the data. The simplifying assumption is consistent with simple, geometrical arguments (the same which apply to the liquid-drop mass formula). The coefficient $K_{\text {Coul }}$ can be calculated. In this way, a value of $K_{\tau}$ equal to $550 \pm 100 \mathrm{MeV}$ has been deduced.

Based on this result, one would be tempted to set another constraint on the Skyrme forces: that is, to select those with the empirical value of $K_{\tau}$. However, a strong warning is appropriate here. Comparing $K_{\tau}$ from the data with the result of Eq. (7) neglects the fact this latter equation does not include any surface-symmetry contribution. We have plotted in Fig. 5 the values of $K_{\infty}$ and $K_{\tau}$ of the 10 selected forces which have been listed at the end of the previous subsection. The shaded box defines the intersection of the constraints on the quantities. Whereas the one on $K_{\infty}$ has been claimed to be robust, the one on $K_{\tau}$ suffers from the mentioned drawback. There is a clear tendency of Skyrme forces to predict values of $K_{\tau}$ which are smaller (in absolute value) than $450 \mathrm{MeV}$. This remains true if a larger sample of Skyrme parametrizations is considered. 


\section{CONCLUSIONS}

While part of the nuclear structure community is striving to construct a universal, accurate energy density functional, many calculations are still performed with e.g. Skyrme forces which should probably be rejected. In fact, there is not consensus on which properties should be necessarily reproduced by a mean-field calculation with an effective force, and what pitfalls should be tolerated.

It is clear that many Skyrme interactions have been built with an eye on very specific applications and should not be used systematically. In this paper, we focus on the performance of Skyrme forces when they are applied to the study of giant resonances, in particular the ISGMR and IVGDR. We do not calculate these modes by using a huge ensemble of Skyrme parameter sets, but we use for the purpose of screening the results of Ref. [4], that is, we demand first that the overall behavior of the energy per particle both in symmetric uniform matter and in neutron matter are reasonable in the sense defined in the quoted work.

The results for the ISGMR and IVGDR in ${ }^{208} \mathrm{~Pb}$ are claimed to be a valid constrain to be imposed on existing forces as well as on envisaged new functionals. This in keeping with the fact that we have shown that the constraints can be translated into conditions on physical parameters which characterize the nuclear EOS like the nuclear matter incompressibility and the symmetry energy at sub-saturation density. We have also demonstrated that it is not straighforward to extend trivially the considerations made for e.g. ${ }^{208} \mathrm{~Pb}$, to the case of open-shell nuclei. In the case of ISGMR, in particular, we have elucidated the role played by the pairing correlations.

The ISGQR could be considered as well as an input for our considerations, whereas in the case of spin and spin-isospin modes probably extensions of the effective forces should be envisaged (besides other reasons, to avoid instabilities of uniform matter in the spin and spin-isospin channels [31]).

\section{ACKNOWLEDGMENTS}

Many of the results reported here have been obtained through collaborations with colleagues and students. In particular, the author would like to thank L. Capelli, J. Li, J. Meng, L. Trippa, E. Vigezzi. Discusssions with U. Garg about the data and the analysis of Ref. [29] are gratefully acknowledged. Thanks are also due to B. Tsang and B.A. Li for providing the author with the data displayed in Fig. 2, and for clarifications about the issue of the symmetry energy extracted from the study of heavy-ion collisions. The authors expresses special thanks to P. Danielewicz for warning against a strict comparison of $K_{\tau}$ from data and from Eq. (7).

\section{REFERENCES}

1. See the contribution of R. Wiringa in this volume.

2. M. Bender, P. H. Heenen, P. G. Reinhard, Rev. Mod. Phys. 75, 121 (2003).

3. See the contribution of M. Pearson in this volume.

4. J. Rikovska Stone, J. C. Miller, R. Koncewicz, M. D. Strayer, Phys. Rev. C 68, 034324 (2003).

5. G. Colò, P. F. Bortignon, S. Fracasso, N. Van Giai, Nucl. Phys. A 788, 173 (2007).

6. J. Li, G. Colò, J. Meng, Phys. Rev. C 78, 064304 (2007).

7. J. Terasaki, J. Engel, M. Bender, J. Dobaczewski, W. Nazarewicz, and M. Stoitsov, Phys. Rev. C 71, 034310 (2005).

8. E. Khan, N. Sandulescu, M. Grasso, and N. Van Giai, Phys. Rev. C 66, 024309 (2002); L. Capelli, G. Colò, J. Li, Phys. Rev. C, submitted.

9. L. Trippa, G. Colò, E. Vigezzi, Phys. Rev. C 77, 061304(R) (2008).

10. L. Trippa, M.Sc. thesis, University of Milano (unpublished).

11. J. P. Blaizot, Phys. Rep. 64, 171 (1980).

12. http: \\www. unedf .org.

13. H. S. Köhler, Nucl. Phys. A 258, 301 (1976).

14. B. K. Agrawal, S. Shlomo, and V. Kim Au, Phys. Rev. C 68, 031304 (2003).

15. L. G. Cao, U. Lombardo, C. W. Shen, and N. Van Giai, Phys. Rev. C 73, 014313 (2006).

16. S. S. Dietrich and B. L. Berman, At. Data Nucl. Data Tables 38, 199 (1988).

17. D. Youngblood, H. L. Clark, and Y. W. Lui, Phys. Rev. Lett. 82, 691 (1999).

18. R. C. Tolman, Proc. Natl. Acad. Sci. U.S.A. 20, 3 (1943); J. R. Oppenheimer and G. M. Volkov, Phys. Rev. 55, 374 (1939).

19. S. L. Shapiro, S. A. Teukolsky, Black holes, white dwarfs, and neutron stars, John Wiley \& Sons, New York, 1983, pp. 125 and 241.

20. A. Klimkiewicz et al., Phys. Rev. C 76, 051603(R) (2007).

21. P. Danielewicz, J. Lee, arXiv:0811.3107. 
22. M. B. Tsang, Y. Zhang, P. Danielewicz, M. Famiano, Z. Li, W. G. Lynch, A. W. Steiner, Phys. Rev. Lett. (in press).

23. L. W. Chen, C. M. Ko, and B. A. Li, Phys. Rev. Lett. 94, 32701 (2005).

24. H. Sagawa, S. Yoshida, X.-R. Zhou, K. Yako, and H. Sakai, Phys. Rev. C 76, 024301 (2007).

25. C. J. Horowitz, J. Piekarewicz, Phys. Rev. Lett. 86, 5647 (2001).

26. S. Shlomo, V. M. Kolomietz, and G. Colò, Eur. Phys. J. A 30, 23 (2006); G. Colò, Physics of Elementary Particles and Atomic Nuclei (PEPAN) 39, 286 (2008).

27. J. Piekarewicz, Phys. Rev. C 76, 031301(R) (2007).

28. D. H. Youngblood, Y.-W. Lui, H. L. Clark, B. John, Y. Tokimoto, and X. Chen, Phys. Rev. C 69, 034315 (2004); Y.-W. Lui, D. H. Youngblood, Y. Tokimoto, H. L. Clark, and B. John, Phys. Rev. C 70, 014307 (2004).

29. T. Li et al., Phys. Rev. Lett. 99, 162503 (2007).

30. U. Garg (private communication).

31. H. Sagawa, J. Margueron (to be published). 